\title{
Ocorrência do delirium nas unidades de terapia intensiva
}

\author{
Larissa Caetano Garnett*, Patricia Goulart Rosa Cardoso, M.Sc.**
}

${ }^{*}$ Enfermeira supervisora de cuidadores de alunos com deficiência, Especialista em Enfermagem em Cuidados Críticos/ Cardiologia pela Universidade do Vale do Paraíba, São José dos Campos/SP, **Enfermeira, Professora pela Universidade do Vale do Paraíba, Parte integrante da comissão de biossegurança da Faculdade de Ciências da Saúde (FCS) da Universidade do Vale do Paraíba, Responsável técnica pela CME e Centro de Práticas Supervisionadas da FCS da Universidade do Vale do Paraíba

\section{Resumo}

Caracterizado pelo curso flutuante e diminuição no nível de consciência, o delirium é considerado uma alteração neurológica que acomete principalmente idosos institucionalizados. Este estudo teve como objetivo buscar nas produçóes científicas a ocorrência da clínica delirium em unidades de terapia intensiva (UTI) e a atuação da enfermagem frente esta síndrome. Foram selecionados 35 artigos utilizando os descritores na língua portuguesa, inglesa e espanhola na base de dados do Google Acadêmico e Biblioteca Virtual em Saúde (BVS), Pubmed, Scientific Eletronic Library Online (Scielo), Medline, Lilacs e Ibecs. Destes $45 \%$ abordam sobre a assistência de enfermagem para pacientes com delirium, 40\% abordam a ocorrência do delirium de pacientes em UTI e 15\% abordando a assistência de enfermagem e ocorrência do delirium em UTI. Dos artigos analisados observou-se que a abordagem descreve sobre a história desta clínica, sua fisiopatologia e diagnóstico médico destacando a elevada incidência e o desconhecimento por parte dos profissionais acerca deste distúrbio que permanece subdiagnosticado. Sobre a assistência de enfermagem, destacamos a não existência de protocolos, condutas de enfermagem, treinamentos ou sugestáo de conduta frente à ocorrência de delirium, permitindo inferir a necessidade de novos estudos no direcionamento da assistência de enfermagem na detecção do delirium.

Palavras-chave: delirium, unidades de terapia intensiva, assistência de enfermagem.

\section{Abstract}

\section{Occurrence of delirium in intensive care units}

Delirium is characterized by fluctuating course and decreasing level of consciousness and is considered a neurological disorder that affects mainly elderly institutionalized patients. This study aimed at searching in scientific productions the occurrence of delirium in medical intensive care units (ICU) and nursing performance facing this syndrome. We selected 35 studies using the key-words in Portuguese, English and Spanish in the database Google Scholar and the Virtual Health Library (VHL), Pubmed, Scientific Electronic Library Online (Scielo), Medline, Lilacs and IBECS. Forty-five percent of 35 studies focus on nursing care for patients with delirium, forty percent discuss the occurrence of delirium in ICU patients and fifteen percent are based on nursing care and occurrence of delirium in the ICU. We observed in these studies that they reported the history of this clinic, its pathophysiology and medical diagnosis, highlighting the high incidence 
and lack of knowledge of some professionals about this disorder which remains underdiagnosed. Concerning nursing care, we verify the lack of protocols, nursing procedures, training or suggestion of procedures to deal with the occurrence of delirium. Further studies designed to detect delirium during nursing care are required.

Key-words: delirium, intensive care unit, nursing care.

\section{Resumen}

\section{Ocurrencia del delirium en unidades de cuidados intensivos}

El delirium es un trastorno neurológico de curso fluctuante, que se caracteriza por alteraciones de conciencia y ocurre principalmente en adultos mayores institucionalizados. Este estudio tuvo como objetivo buscar en las producciones científicas la ocurrencia de delirium en unidades de cuidados intensivos (UCI) y la actuación de enfermería ante este síndrome. Fueron seleccionados 35 estudios utilizando los descriptores de lengua portuguesa, inglesa y española en la base de datos del Google Académico y Biblioteca Virtual en Salud (BVS), Pubmed, Scientific Eletronic Library Online (Scielo), Medline, Lilacs e Ibecs. Un 45\% aborda el cuidado de enfermería para pacientes con delirium, un $40 \%$ la ocurrencia del delirium en UCI y un 15\% la atención de enfermería y la ocurrencia de delirium en UCI. En los estudios analizados se observó que el abordaje describe la historia de esta clínica, su fisiopatología y diagnóstico médico destacando la elevada incidencia y desconocimiento por parte de los profesionales acerca de este disturbio que permanece subdiagnosticado. Con referencia al cuidado de enfermería, señalamos la inexistencia de protocolos, de conductas de enfermería, de entrenamiento o de sugestión de conducta ante la ocurrencia de delirium. De esto se infiere la necesidad de nuevos estudios dirigidos a la atención de enfermería para la detección del delirium.

Palabras-clave: trastornos de delirio, unidades de cuidados intensivos, atención de enfermería.

\section{Introdução}

As unidades de terapia intensiva (UTI) são consideradas setores de alta complexidade direcionados a pacientes gravemente enfermos que necessitam de cuidado intensivo. Descritas como geradores de inseguranças, tensões e medos são capazes de promover transtornos psicológicos nos pacientes que ali permanecem, dentre eles o delirium [1].

O delirium é considerado um distúrbio neurológico de início abrupto caracterizado por diminuição das funções cognitivas, redução no nível de consciência e desordem no ciclo sono vigília, podendo levar o doente a ficar diversas noites sem dormir [2]. Sua ocorrência gira em torno de 80,0\% dos pacientes internados na UTI sendo mais comum entre pacientes idosos, sob ventilação mecânica e que oscilam entre estágios de hiperatividade e hipoatividade. Estimativas mostram que em 2050, 25,0\% da população será idosa, portanto, estudar doenças predominantes desta faixa etária é de fundamental importância [3].

A relevância do estudo da ocorrência do delirium não se deve somente à elevada incidência, mas, sobretudo, às suas consequências dentre as quais destacamos o aumento significativo das taxas de morbidade e mortalidade, maior dificuldade em realizar o desmame ventilatório, prolongamento do período de tratamento e aumento de seu custo, o comprometimento da recuperação global do indivíduo e possíveis sequelas em longo prazo [4].

Autores referem não ser possível eleger ao certo os fatores predisponentes ao desenvolvimento deste transtorno que pode ser desencadeado por fatores como idade avançada e consequentes alteraçôes em neurotransmissores, déficit visual e auditivo prévio, restrição no leito, desnutrição grave, uso de sedaçáo continua por um longo período, uso de determinadas medicaçóes e acompanhados por uma grave doença de base. Para avaliar o grau de agitação e sedação foi elaborada a escala de sedação e agitação de Richmond (RASS), que avalia o paciente numericamente e o Confusion Assessment Method (CAM) que se trata de um modelo internacional para análise do delirium [5].

O diagnóstico é realizado pelo médico através de observação minuciosa dos sintomas, no entanto verifica-se a necessidade de estudos que possibilitem o reconhecimento deste quadro, inclusive pela equipe de enfermagem que permanece constantemente ao lado do cliente durante todo o período de internação. 
Autores dividem o tratamento em três etapas, sendo elas: preventiva, com tratamento dos fatores desencadeantes e cuidados com intuito de reverter alguns sintomas já existentes. É importante que o ambiente seja adequado a manutenção da orientação do paciente permitindo visualização da luz natural, horário, preservação do sono e evitar contençóes prolongadas, estimular a permanência da família ao lado do paciente sempre que possível assim como cuidados de suporte analgesia correta e controle de eliminaçóes fisiológicas são fatores importantes para manutenção da hidratação adequada. $\mathrm{O}$ controle farmacológico é indicado nos casos em que o paciente representa risco a si mesmo e aos demais não havendo consenso sobre a medicação a ser utilizada [6].

\section{Material e métodos}

Trata-se de um estudo desenvolvido a partir do método descritivo exploratório com recorte temporal retrospectivo baseado em artigos científicos com enfoque em terapia intensiva e ocorrência de delirium. A realização deste estudo foi embasado em publicaçóes cientificas nos idiomas português, inglês e espanhol com recorte temporal retrospectivo abrangendo período entre 2000 e 2013. Os periódicos foram selecionados nos bancos de dados do Google Acadêmico e Biblioteca Virtual em Saúde (BVS), Pubmed, Scientific Eletronic Library Online (Scielo), Medline, Lilacs e Ibecs. Os descritores utilizados foram: delirium em UTI, enfermagem e delirium em UTI, assistência de enfermagem em UTI. Para a realização da coleta de dados foi elaborado um formulário para registro dos artigos selecionados e um segundo formulário para análise das temáticas. Os resultados foram analisados através de percentual simples.

\section{Revisão de literatura}

Criadas com o intuito de oferecer suporte a pacientes graves as UTI são setores peculiares com fatores intrínsecos que podem gerar alto grau de stress aos pacientes e seus familiares podendo causar efeitos negativos pelo excesso de luz, ruído, isolamento e gravidade dos pacientes que ali se encontram. Pesquisas referem que estes fatores são causadores de comorbidades como o delirium e que podem ser minimizados pelo cuidado humanizado por parte dos profissionais oferecendo não apenas clínico mas também cuidado terapêutico e integral reduzindo fatores agravantes [7].

A síndrome do Delirium é descrita como agitação psicomotora ou hipoatividade e incapacidade de manter contato com o ambiente externo, diferencia-se de confusáo mental por ter curso flutuante e fatores predisponentes característicos, podendo ser observada principalmente em pacientes com longa permanência em UTI [8]. Tais sintomas podem ser observados em uma incidência de 14,0 a $56,0 \%$, sendo ainda maior se pesquisado apenas em pacientes idosos, fator que agrava a probabilidade da ocorrência e evolução da síndrome [9].

Autores ressaltam que o delirium causa perda da capacidade funcional podendo em longo prazo acarretar a inserção de idosos pelos seus familiares em instituiçóes de longa permanência. A causa permanece desconhecida sendo a hipótese mais aceita a de que ocorra devido a anormalidades na produção e liberação de neurotransmissores responsáveis pelo comportamento e cognição como acetilcolina, noradrenalina, serotonina, gaba e dopamina sendo na maioria das vezes um marcador de disfunção orgânica [10].

Estudiosos corroboram quanto à importância do diagnóstico precoce feito apenas através de sinais e sintomas percebidos principalmente pela equipe de enfermagem durante os cuidados prestados ao cliente. Como há uma intensa relação entre fatores predisponentes e pacientes vulneráveis é fundamental a detecção de distúrbios mentais prévios a internação hospitalar, retirada abrupta de medicações de uso prolongado como sedativos, déficit visual e/ ou auditivo além de agravantes como a longa permanência em UTI sem possibilidade de orientação temporal, contençáo mecânica prolongada no leito, privação do sono dentre outros [11].

Para Costa e Marcon 2009 [12] o trabalho da enfermeira de UTI possui características particulares, deve aliar a tecnologia empregada para assistir os pacientes de forma permanente com humanização no cuidado assumindo uma posição ética e de respeito. Apesar de o trabalho poder ser avaliado individualmente, o resultado somente será satisfatório se a equipe trabalhar em harmonia e equilíbrio através de uma eficiente supervisáo, portanto a dinâmica e a relação entre os colaboradores podem gerar mudanças de atitudes e comportamentos. Considerando que durante a internação na maioria das vezes o paciente encontra-se completamente dependente da equipe de enfermagem o cuidado prestado in- 
fluenciará diretamente sobre a saúde física e mental do cliente. Salicio e Gaiva [13] possuem semelhantes opiniôes em relação à humanização e qualidade na assistência não sendo dissociáveis quando o intuito é oferecer a possibilidade de reabilitação ao paciente grave, porém, recuperável [14].

\section{Resultados e discussão}

Para a realização desta pesquisa 35 artigos foram selecionados, observando-se grande escassez de publicaçóes acerca dos cuidados de enfermagem frente ao paciente com delirium. Onze destas pesquisas foram realizadas por profissionais da área médica sendo a grande maioria destas sobre o diagnóstico e tratamento e apenas sete artigos foram publicados por enfermeiros.

Para os descritores delirium em UTI foram selecionados 14 artigos, representando $40 \%$ do total, que abordam a perspectiva histórica do delirium, a realizaçáo do diagnóstico em UTI e sua fisiopatogenia. Cinco pesquisas (15\%) publicadas nos últimos cinco anos, referem-se à enfermagem e o delirium em UTI destacando o diagnóstico de enfermagem, anotaçóes produzidas pela equipe frente ao delirium e o despreparo desta classe frente esta clínica.

Com maior número de pesquisas publicadas, a temática assistência de enfermagem em UTI, artigos relevantes à abordagem generalizada do cuidado foram selecionados totalizando 15 artigos (45\%) selecionados para este estudo. Em sua maioria os artigos discorrem sobre as tecnologias utilizadas em UTI, a percepção do paciente e da família acerca deste setor e os cuidados de enfermagem a fim de minimizar os impactos causados.

Existe uma prevalência de publicações disponíveis que ressaltam apenas a atuação da equipe médica frente ao controle da dor, sedação e analgesia para minimizar os riscos da ocorrência do delirium. Em contrapartida pesquisadores revelam que ainda assim os profissionais da enfermagem são os que mais relatam nos prontuários os distúrbios neurológicos sendo que a implantação do instrumento CAM-ICU facilitou a comunicação interdisciplinar [15].

Dentre os relatos de anotaçóes de enfermagem os resultados deste estudo apontam para um predomínio sobre condutas como a contenção mecânica do paciente que se encontra agitado. Observou-se a falta de conhecimento, de planejamento e detecção de características como curso flutuante da síndrome diferenciando-a de outros distúrbios, pela equipe de enfermagem. Por outro lado notou-se que o diagnóstico médico em 25,0 a $75 \%$ dos casos não são realizados corretamente podendo chegar a $100 \%$ se o delirium se apresentar na forma hipoativa, podendo ser facilmente confundido com depressão [16].

Pesquisadoras ressaltam a necessidade da elaboração do diagnóstico de enfermagem pelo enfermeiro conforme resolução do Conselho Federal de Enfermagem (COFEN) construindo o processo eficaz de cuidado individualizado ao paciente. Não foram encontrados artigos que comprovem a importância da detecçáo precoce da síndrome pela equipe de enfermagem, apesar de estes profissionais poderem alterar o curso da doença mantendo atitudes simples como diminuição de ruídos, evitar contenção mecânica, manter paciente orientado quanto ao tempo e ao espaço, permitir a presença da família sempre que possível ao lado do cliente criando um vínculo do mesmo com o meio exterior [7].

Figura 1 - Artigos selecionados de acordo com os

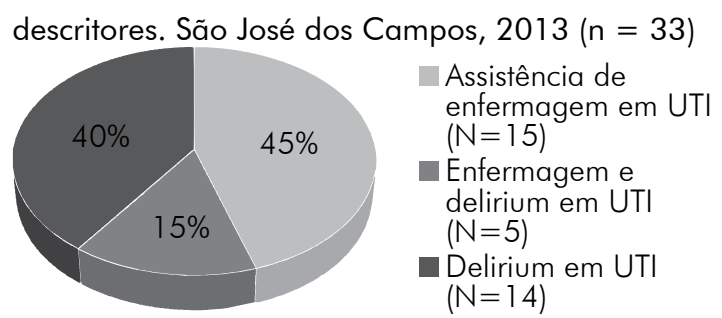

\section{Conclusão}

As publicações acerca do tema delirium são recentes e neste estudo observou-se que a maioria dos artigos analisados foi publicada por equipe médica que referendaram a importância desta clínica, destacaram a frequência da ocorrência e que muitos pacientes não recebem o diagnóstico (subdiagnóstico) e tratamento corretamente.

$\mathrm{Na}$ análise das publicaçôes científicas observou-se o registro de ocorrência do delirium ocorrer em $14 \%$ a $56 \%$ dos pacientes idosos institucionalizados, e que em mais de $67 \%$ dos casos o diagnóstico não é feito de maneira correta.

São frequentes relatos sobre a importância da equipe de enfermagem frente à detecção precoce do delirium em unidades de terapia intensiva, porém não há relatos de estudos que comprovem este beneficio. Também se observou neste estudo que a falta de conhecimento dos profissionais de saúde na detecção precoce desta clínica (tanto da enfermagem 
como de médicos) geram condutas que podem agravar a ocorrência de delirium.

\section{Referencias}

1. Souza AD, Lima CGG, Franco LMC, Berçante TA. Instrumentos de gestáo para avaliaçáo da carga de trabalho da enfermagem em unidades de terapia intensiva. Revista Enferm UFPE 2010;4(1):333-40.

2. Cruz JN, Tomasi CD, Alves SC, Macedo RC, Giombelli V, Cruz JGP, et al. Incidência de delirium durante a internaçâo em unidade de terapia intensiva em pacientes pré-tratados com estatinas no pós-operatório de cirurgia cardíaca. Rev Bras Ter Intensiva 2012;24(1):52-7.

3. Silva RFLC, Moreira LR. Fatores de risco para ocorrência de delirium em idosos em terapia intensiva. Revista Enfermagem 2012;15(1):102-21.

4. Salluh JI, Pandharipande P. Prevenção do delirium em pacientes críticos: um recomeço? Rev Bras Ter Intensiva 2012; 24(1):1-3.

5. Fernandes CR, Gomes JMA, Moraes RP, Marinho DS, Holanda MA, Oliveira MRD. Avaliação sistemática do delirium e da dor em pacientes criticamente enfermos. Rev Dor 2009;10(2):158-68.

6. Santos FS, Babichack AC, Amaral A. Delirium em pacientes idosos. Revista Bras Med 2010;57(10).

7. Mori S, Kashiba IK, Silva DV, Zanei SSV, Whitaker IK. Confusion assessment method para analisar delirium em unidade de terapia intensiva. Revisão de literatura. Rev Bras Ter Intensiva 2009;21(1):58-64.
8. Wacker P, Nunes PV, Forlenza V. Delirium: uma perspectiva histórica. Revista de Psiquiatria Clínica 2005;32( 3): 97-103.

9. Coulson BS, Almeida OP. Delirium: moving beyond the clinical diagnosis. Rev Bras Psiquiatr 2002; 24(1): 28-33.

10. Coelho TD, Machado FS, Joaquim MAS. Delirium em Terapia Intensiva: Fatores de Risco e Fisiopatogenia. Revista Portuguesa de Terapia Intensiva 2011;18(3):5282.

11. Silva CL, Firmino JS, Knopfholz J, Roznowski KC. Delirium: emergência clinica de difícil diagnóstico e os cuidados de enfermagem aos pacientes. Publicaçáo UEPG 2011;17(2):91-7.

12. Costa JB, Marcon SS. Elaboraçáo de um instrumento para identificar memórias referentes à Unidade de Terapia Intensiva. J Bras Psiquiatr 2009;58(4)223-30.

13. Salicio DMBS, Gaiva MAM. O significado de humanização da assistência para enfermeiros que atuam em UTI. Rev Eletr Enf 2006;8(3):370-6.

14. Silva IAS, Cruz EA. Trabalho da enfermeira intensivista: um estudo da estrutura das representaçóes sociais. Rev Esc Enferm USP 2008;42( 3):554-62.

15. Lourenço EC, Neves EP. As necessidades de cuidado e conforto dos visitantes em UTI oncológica: Uma proposta fundamentada em dados de pesquisa. Rev Bras Cancerol 2008;54(3):213-20.

16. Silva RCG, Paiva AAP, Marques PAO. Análise dos registros produzidos pela equipe de saúde e da percepção dos enfermeiros sobre os sinais e sintomas de delirium. Rev Latinoam Enferm 2011;19(1):81-9. 